\title{
Time Decay of Solutions of Coupled Maxwell-Klein-Gordon Equations
}

\author{
Deivy M. Petrescu ${ }^{\star}$ \\ Departamento de Matemática Aplicada, IME-USP, Brasil. email:deivy@ime.usp.br
}

Received: 28 July 1994/Accepted: 6 October 1995

\begin{abstract}
We obtain the optimal time decay of the solutions of the coupled Maxwell-Klein-Gordon equations in four dimensional spacetime, provided the initial data are what we define as Coulomb. In other words, the initial data are such that the Klein-Gordon field is smooth and compactly supported and the Maxwell field is electrostatic outside this support. The problem involves charge, therefore, the initial data do not satisfy either fast decay or any smallness condition. In spite of that, we are able to obtain our result using the inversion map of the lightcone of a carefully selected origin. We thus, avoided the blow-up that takes place when using the usual conformal transformation to the Einstein spacetime.
\end{abstract}

\section{Introduction}

The presence of charge imposes precise conditions on the decay of the initial electromagnetic field preventing it from being placed in a weighted Sobolev space. Therefore, the usual techniques of conformal transformation or smallness of initial data to prove decay do not work.

The condition that initially the electromagnetic field outside the (compact) support of the Klein-Gordon field is static, permits us to use the inversion map at the lightcone of a carefully selected origin. The conformal covariance of the equations involved and the global existence theorem (Theorem 1) allow us to obtain the optimal time decay for the solutions. This method can not be used with a non-Abelian gauge group because the commutation relation would prevent the fields from being static (the commutators would behave like a source with non-compact support).

In the usual conformal compactification of Penrose-Christodoulou, the generator of the transformation is a conformal Killing field of the Minkowski metric in the whole of spacetime. In contrast, the generator of the inversion is a conformal Killing field in the interior of the lightcone at the point on which the inversion is made. Thus, while with the Penrose-Christodoulou method we have to take into account

\footnotetext{
Work partially supported by FAPESP and CNPq
} 


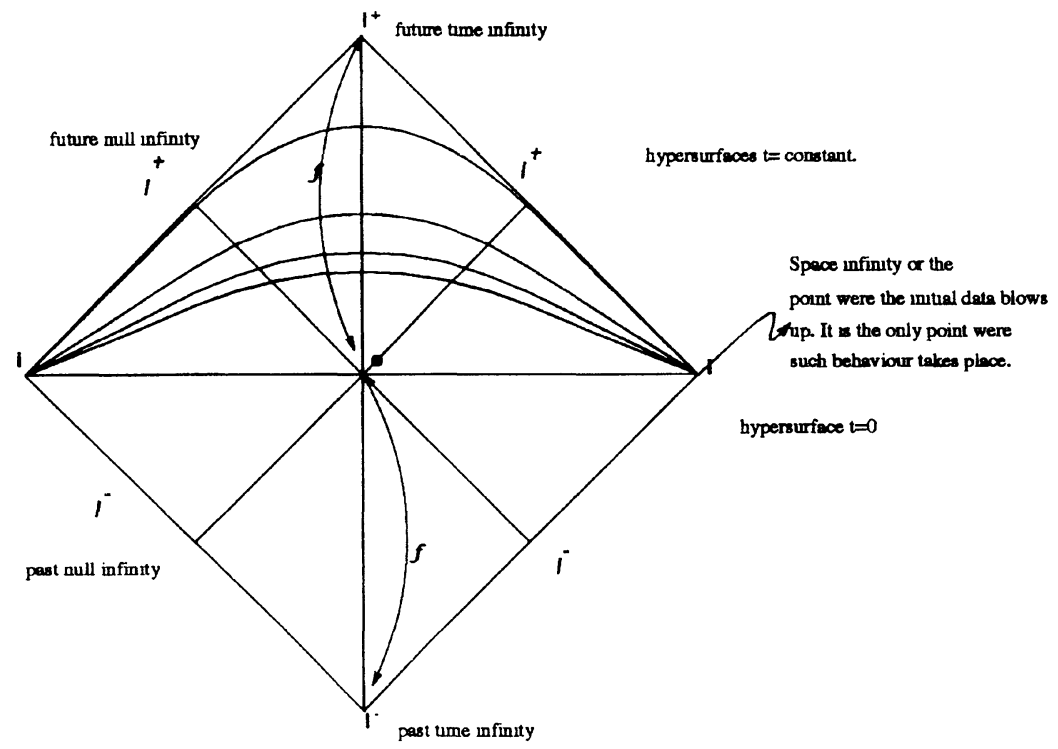

Fig. 1. The inversion map as seen from the Einstein Universe. Observe that the singular point lies in the region complimentary to the one we are working with. The initial data, close to the backwards cone from $\mathrm{I}^{+}$inside the region of interest, is always regular

global behavior of the fields, the space infinity, $\mathbf{i}$, the point where the conformal data blow up, is discarded in the inversion map. (see Fig. 1).

Some previous results for similar problems are the global existence for the YangMills field (see [1,2]), the optimal time decay for the Yang-Mills field with small data (see [5]) and the optimal time decay for the coupled Yang-Mills-Higgs-Spinor fields with small data, where the positivity of the Energy is not necessary (see [4]).

This work is divided as follows, in the next section we explain what is meant by a Coulomb initial data. We show what these data imply in terms of solution. Next, using a global existence theorem, we prepare the grounds for the Cauchy problem in the target space. In Sect. 3 we study the inversion map in detail and discuss some properties of our set of equation vis-a-vis conformal transformations. In Sect. 4 we establish the regularity of the Cauchy data in the target space allowing us to use the global existence theorem. In the last section, we obtain the optimal time decay of the solution.

\section{The Cauchy Problem}

Maxwell equations give rise to a gauge field, the coupling with a scalar field is through the covariant derivative of the scalar field in the principal bundle (minimal coupling). The Lagrangian for this problem is

$$
\mathscr{L}=\eta^{\alpha \beta} \eta^{\mu v} F_{\alpha \mu} \cdot F_{\beta v}-D_{\beta} \phi \cdot D^{\beta} \phi,
$$

where $\eta_{\mu v}=\operatorname{diag}(-,+,+,+)$ and $D_{v}=\partial_{v}+i A_{v}, \phi$ is a complex function, $\cdot$ is a gauge invariant scalar product given by $A \cdot B=\operatorname{Re}(A \bar{B})$, where $A$ and $B$ are complex functions and the $\overline{\mathrm{bar}}$ denotes the usual complex conjugation. 
The equations of motion are the Maxwell-Klein-Gordon equations. They are

$$
\begin{aligned}
\partial_{\alpha} F_{\mu \nu}+\partial_{\mu} F_{v \alpha}+\partial_{\nu} F_{\alpha \mu} & =0 \\
\partial_{v} F^{v \mu} & =\operatorname{Im}\left(\phi \overline{D^{\mu} \phi}\right), \\
D^{v} D_{v} \phi & =0,
\end{aligned}
$$

or using a different notation

$$
\begin{aligned}
\mathbf{d F} & =0, \\
\delta \mathbf{F} & =\mathbf{J}, \\
\square \phi & =-i\left(2 A^{v} \partial_{v} \phi+\phi \partial_{v} A^{v}\right)+A_{v} A^{v} \phi_{j},
\end{aligned}
$$

where $\mathbf{F}$ is the 2-form electromagnetic tensor, $\mathbf{A}$, the 1-form electromagnetic potential $\mathbf{F}=\mathbf{d A}$ and $\mathbf{J}=\operatorname{Im}\left(\phi \overline{D_{\mu} \phi}\right)$ is the 1-form, 4-charge-density. The gauge group is $\mathbf{U}(1) . \square$ is the wave operator (D'Alembertian), $\mathbf{d}$ is the exterior differential operator, $\delta=*^{-1} \mathbf{d} *$ is the degree -1 differential operator and $*$ is the Hodge dual. The time component of Eq. (3) is known as Coulomb's law

$$
\nabla \mathbf{E}=\operatorname{Im}\left(\phi \overline{\partial_{0} \phi}\right)
$$

an elliptic equation which imposes a time independent constraint on the electric field. In the region outside the support of $\phi$ the electromagnetic field satisfies

$$
\begin{aligned}
\mathbf{d F} & =0, \\
\delta \mathbf{F} & =0 .
\end{aligned}
$$

We say that an initial data set $\left(A_{i}\left(t_{0}, x\right), E_{i}\left(t_{0}, x\right)=F_{i 0}\left(t_{0}, x\right), \phi\left(t_{0}, x\right), \partial_{0} \phi\left(t_{0}, x\right)\right)$ for the Maxwell-Klein-Gordon equations (2-4) is a Coulomb initial data iff

- (h1) $\mathbf{A}$ is given in the temporal gauge, i.e., $A_{0}(t)=0$.

- (h2) The scalar (charge-density) field $\phi\left(t_{0}, x\right)$ is smooth, supported in a ball of radius $r_{0}$ around the origin, i.e., $\phi\left(t_{0}, x\right) \in C_{0}^{\infty}\left(B_{r_{0}}\right)$.

- (h3) For any $r=|\mathbf{x}|>r_{0}$, the electric and the electromagnetic potential field are given by

$$
\begin{aligned}
& \mathbf{E}\left(t_{0}, x\right)=\nabla \int_{\mathscr{V}} \frac{\operatorname{Im}\left(\phi\left(x^{\prime}\right) \overline{\partial_{0} \phi\left(x^{\prime}\right)}\right)}{\left|\mathbf{x}-\mathbf{x}^{\prime}\right|} d^{3} x^{\prime}, \\
& \mathbf{A}\left(t_{0}, x\right)=t_{0} \cdot \mathbf{E}(x)
\end{aligned}
$$

where the electric field given by Eq. (11) is the field produced outside the support of a charge distribution with charge-density $\rho(x)=\operatorname{Im}\left(\phi(x) \overline{\partial_{0} \phi(x)}\right)$. The potential given by Eq. (12) is unique up to an additive constant.

One writes the radial and angular components of the electrostatic field around the origin as

$$
\begin{aligned}
\mathbf{E}_{\mathbf{r}}(\mathbf{x}) & =\nabla_{r} \int_{\mathscr{V}} \frac{\rho\left(x^{\prime}\right)}{\left|\mathbf{x}-\mathbf{x}^{\prime}\right|} d^{3} x^{\prime}, \\
\mathbf{E}_{\omega}(\mathbf{x}) & =\nabla_{\omega} \int_{\mathscr{V}} \frac{\rho\left(x^{\prime}\right)}{\left|\mathbf{x}-\mathbf{x}^{\prime}\right|} d^{3} x^{\prime},
\end{aligned}
$$


$\omega$ denotes angular coordinates, $r=|\mathbf{x}|, \mathscr{V}$ is the volume enclosed by the ball of radius $r$, keeping in mind that $\rho(x)=\operatorname{Im}\left(\phi(x) \overline{\partial_{0} \phi(x)}\right)$.

Lemma 1. The field given by Eqs. $(13,14)$ satisfies $(9,10)$. In other words, Eqs. $(13,14)$ represent the solution of Maxwell-Klein-Gordon system given by Eqs. (57) outside $\mathbf{B}_{\left(\mathbf{t}-\mathbf{t}_{o}\right)+\mathbf{r}_{0}}$, for all times.

Proof. Outside the support, Eqs. (5-7) reduce to Eqs. (9-10). These, are written as

$$
\begin{gathered}
\nabla \cdot \mathbf{E}=\mathbf{0}, \\
\nabla \times \mathbf{E}=\mathbf{0} .
\end{gathered}
$$

Equation (15) follows from the analyticity of $1 /\left|\mathbf{x}-\mathbf{x}^{\prime}\right|$ in Eqs. $(13,14)$ for $|\mathbf{x}|>\left|\mathbf{x}^{\prime}\right|$. Equation (16) follows from a well known vector identity. The finite speed of propagation of the wave, assures us of the global result.

We are in position to use the following global existence theorem (see $[1,2])$

Theorem 1. If $u_{0}=\left(A_{i}, E_{i}, \phi, \partial_{0} \phi\right)$ is initial data lying in $\left(H_{2+k} \times H_{1+k}\right)^{2}$ for $k \geqq 3$ and satisfying the initial value constraint,

$$
\partial_{i} E_{i}=\operatorname{Im}\left(\phi \overline{\partial_{0} \phi}\right)
$$

then there is a unique solution $u(t) \in\left(H_{2+k} \times H_{1+k}\right)^{2}$ of the temporal gauge Maxwell-Klein-Gordon equations defined for all $t \in(-\infty, \infty)$ and having $u(0)=u_{0}$. The corresponding fields $\left(A_{\mu}(x), F_{\mu \nu}(x), \phi(x), D_{\alpha} \phi(x)\right)$ are globally defined in Minkowski space, lie in $\left(C^{k} \times C^{k-1} \times C^{k} \times C^{k-1}\right)$ and satisfy Eqs. (5-7) in the classical sense.

We use the previous theorem as follows, let us assume that the initial data are given at a time $t_{0}>2 r_{0}+1$, then the support of $\phi$ is contained in the interior of the future directed light cone of the origin. Since it has been established that the solution exists for all times, one can speak of the solution at a later time $t_{1}$, say. Inside the outward future directed lightcone of the origin the solution between $t_{0}$ and $t_{1}$ is finite and regular. In this region we make the following gauge transformation $U: \mathbf{A} \rightarrow \tilde{\mathbf{A}}, \tilde{\mathbf{A}}$ satisfying $\tilde{A}_{0}+\tilde{A}_{r}=0$ with the angular components unchanged. We show that the above transformation is a bonafide gauge transformation. We have

$$
\begin{gathered}
\tilde{A}_{\mu}=A_{\mu}+i U \cdot \partial_{\mu} U^{-1}, \\
U\left(r, t \geqq t_{0}\right) \in \mathbf{U}(1) .
\end{gathered}
$$

Since $\mathbf{A}$ was initially in the temporal gauge we have

$$
\begin{aligned}
-A_{r} & =i U \cdot \partial_{r} U^{-1}+i U \cdot \partial_{0} U^{-1}, \\
& =i U \cdot \partial_{s} U^{-1}, \\
U(0) & =U_{0},
\end{aligned}
$$

where $U=U(s)$ and $s$ is a parameter along lines $t+r=$ constant and $U_{0}$ is such that at $t=t_{0}$ the gauge condition is satisfied. 
$A_{r}$ is smooth and real, by taking its conjugate value in Eq. (19) and using the property of the scalar product, it follows that

$$
\partial_{s} U \bar{U}=0
$$

or, the length of $U$ is a constant of motion. The standard existence theorems for the linear ordinary differential equation tells us that there exists a unique and smooth solution $U(s)$ defined throughout the region $\bigcup_{t=t_{0}}^{t_{1}} \bar{B}_{t}(t)$. Furthermore, since initially $U \in \mathbf{U}(1)$, then, $U \in \mathbf{U}(1)$ for all points of $\bigcup_{t=t_{0}}^{t_{1}} \bar{B}_{t}(t)$. $\tilde{\mathbf{A}}$ satisfies the gauge condition.

The need for the existence of a solution up to a time $t_{1}>t_{0}$ and the change of gauge will be clarified in the next section. There, we show that inversion maps a hypersurface $t=$ constant onto a hyperboloid to the past of the space origin of the hypersurface $-\frac{1}{t}=$ constant in the target space. If we want to know the Cauchy data on a hypersurface in the target space $\underline{t}=$ constant (underline denotes functions in the target space) we must know the values of the solution to the future of $t_{0}$. The gauge selected above is such that $\mathbf{A}$ is mapped smoothly onto such hyperspace.

\section{The Inversion Map}

Before we discuss the inversion map we prove the following proposition concerning conformal transformations

Proposition 1. Let $M, \eta$ be a four dimensional manifold where $\eta$ is Lorentzian metric, then for $h:(M, \eta) \rightarrow(M, g), g=\Omega^{2} \eta(\Omega>0)$ a conformal automorphism, together with the transformation $h: \phi \rightarrow \Phi=\Omega^{-1} \phi$ the set of Eqs. (2-4) and (9-10) are conformally covariant.

Proof. Invariance of Eq. (2) follows since it is independent of the metric. To prove the covariance of Eq. (3) one uses well known relations for conformal transformations (see [4]) to obtain

$$
\underline{\nabla}_{\alpha} \underline{g}^{\alpha v} \underline{g}^{\beta \mu} F_{v \mu}=\Omega^{-4} \nabla_{\alpha} F^{\alpha \beta},
$$

for the field. Using that $\underline{J}_{\alpha}=\operatorname{Im}\left(\Phi \bar{D}_{\alpha} \Phi\right)$, since $\Phi$ and not $\phi$ is defined in the target space, one has for the current

$$
\underline{g}^{\alpha \beta} \underline{J}_{\alpha}=\Omega^{-2} \underline{J}^{\beta}=\Omega^{-2} \Omega^{-2} \operatorname{Im}\left(\phi \overline{D^{\beta} \phi}\right)=\Omega^{-4} J^{\beta} .
$$

This establishes the desired result. The covariance of Eqs. $(9,10)$ follows, since in this case $\phi=0$.

To prove that the coupled Klein-Gordon equation is conformally covariant, one uses the well known covariance of the operator $\square-\frac{(n-1)}{4 n} \mathscr{R}$ applied to a function to get in the four dimensional case

$$
\begin{aligned}
\Omega^{3}\left(\square_{I} \Phi-\frac{1}{6} \mathscr{R} \Phi\right) & =\Omega^{3} g^{\nu \mu}\left[A_{\mu} A_{v} \Phi-i\left(2 A_{\mu} \partial_{\nu} \Phi+\Phi \partial_{\mu} A_{\nu}\right],\right. \\
\square_{I} \Phi-\frac{1}{6} \mathscr{R} \Phi & =g^{v \mu}\left[A_{\mu} A_{v} \Phi-i\left(2 A_{\mu} \partial_{\nu} \Phi+\Phi \partial \mu A_{v}\right)\right] .
\end{aligned}
$$

$\square_{I}$ is the covariant D'Alembertian and $\mathscr{R}$ the scalar curvature in the image space. We obtained the desired covariance. 
Denote by $C_{0}^{+}$the interior of the future directed lightcone of the origin, i.e., $r^{2}-t^{2}<0, t>0$ and by $C_{0}^{-}$the interior of the past directed lightcone at the origin, i.e., $r^{2}-t^{2}<0, t<0$. Let $\mathscr{C}$ be the mantle of a cone and let $S_{\phi(t)}$ be the support of $\phi$ at time $t$. We shall consider in this section the following conformal automorphism of $C_{0}^{+} \cup C_{0}^{-}$onto itself

by

$$
f: C_{0}^{+} \cup C_{0}^{-} \rightarrow C_{0}^{+} \cup C_{0}^{-}
$$

$$
\underline{x}_{v}=f(x)=\frac{\eta_{\nu \mu} x^{\mu}}{-\eta_{\gamma \beta} x^{\gamma} x^{\beta}},
$$

where coordinates of the image space are underlined. Since $g=f * \eta(f(x))=\Omega^{2} \eta$, where $\Omega^{-1}(x)=-x \cdot x=t^{2}-r^{2}=-s^{2}(x), f$ is said to be a conformal map from $C_{0}^{+} \cup C_{0}^{-}$onto itself $^{1} . f$ is called inversion map. The map $f$ preserves the null structure (i.e., maps null surfaces into null surfaces). Since $\Omega^{2}>0, f$ maps future directed timelike vectors into future directed timelike vectors.

The line element in $M^{+}$, the region $t \geqq 0$ in the Minkowski spacetime, is given by

$$
d s^{2}=-d t^{2}+d r^{2}+r^{2} d \omega^{2},
$$

$\omega$ being angular coordinates. The transformation of coordinates for $t$ and $r$ are

$$
\begin{gathered}
\underline{r}=\frac{1}{2}(\underline{v}+\underline{u})=-\frac{v+u}{2 u v}=\frac{-r}{u v}, \\
\underline{t}=\frac{1}{2}(\underline{v}-\underline{u})=\frac{v-u}{2 u v}=\frac{t}{u v}
\end{gathered}
$$

with the same angular coordinates. Then

$$
d \underline{s}^{2}=\frac{1}{\left(t^{2}-r^{2}\right)^{2}}\left(-d t^{2}+d r^{2}+r^{2} d \omega^{2}\right) .
$$

If one uses null coordinates $u=r-t, v=t+r$ and notices that $s^{2}=u v$, then

$$
d \underline{s}^{2}=\frac{d u}{u^{2}} \frac{d v}{v^{2}}+\frac{(v+u)^{2}}{(2 v u)^{2}} d \omega^{2}
$$

or

$$
\begin{aligned}
& d \underline{u}=\frac{d u}{u^{2}} \rightarrow \underline{u}=\frac{-1}{u}, \\
& d \underline{v}=\frac{d v}{v^{2}} \rightarrow \underline{v}=\frac{-1}{v} .
\end{aligned}
$$

Thus along the $t$ axis, the points $a$ and $b$ such that $t_{a}<t_{b}$ are transformed to points such that $\underline{t}_{a}<\underline{t}_{b}$ (but $\left|\underline{t}_{a}\right|>\left|\underline{t}_{b}\right|$ ). So, future directed nullcones in $C_{0}^{+}$are mapped onto future directed bounded nullcones in $C_{0}^{-}$.

Because $\Omega(x)=-\underline{s}^{-2}(x)=-s^{2}(x)$, we see that $f^{2}=i d$. Then, $f$ maps $\mathscr{C}_{0}^{+}$ onto $\mathscr{C}_{I^{-}}^{+}$and vice versa. $\mathscr{C}_{0}^{-}$in the image spacetime bounds $M^{+} . \mathscr{C}_{0}^{-}$is the image of $\mathscr{C}_{I^{+}}^{-}$of $M^{+}$(see Fig. 1).

\footnotetext{
${ }^{1} f$ is not conformal if we consider the whole of Minkowski space
} 
The map $f$ does not deal with the region outside $\bar{C}_{0}^{+}$, the closure of $C_{0}^{+}$. It is precisely in this region that the energy blows up. By assumption, the solution at any point outside $\bar{C}_{0}^{+}$is explicitly obtained using Lemma 1 . This is why we use the inversion map. The map to Einstein space is a compactification of the whole Minkowski space, it takes into account the global behavior of the initial data, including $\mathbf{i}$, the point at space infinity.

If we prove that the solution up to the time $t_{1}$ in $\bar{C}_{0}^{+}$can be mapped to a regular "initial" data on a spacelike hypersurface $\underline{t}=$ constant in $\bar{C}_{0}^{-}$, then, using the global existence theorem for Maxwell-Klein-Gordon equations we establish existence of solutions up to the origin in the target space.

We have to study what happens to the image of the hypersurface $t=$ constant of the original spacetime. Thus, we look at

$$
t_{0}=\frac{\underline{t}}{\underline{r}^{2}-\underline{t}^{2}} \quad t_{0} \text { constant }
$$

Working it out, we get

$$
\begin{gathered}
\underline{r}^{2}-\underline{t}^{2}=\frac{\underline{t}}{t_{0}}, \\
\underline{r}^{2}-\left(\underline{t}+\frac{1}{2 t_{0}}\right)^{2}=-\frac{1}{4 t_{0}^{2}},
\end{gathered}
$$

that is, points of the plane $t=$ constant are mapped into the hyperboloid (33), and for $\underline{r}=0$ this hyperboloid crosses the $t$ axis at $\underline{t}=-\frac{1}{t_{0}}$. Some simple computations show that choosing

$$
t_{1}=\frac{\left(t_{0}-r_{0}\right)^{2}}{\left(t_{0}-2 r_{0}\right)}
$$

the image of the support of $\phi$ at $t=t_{1}$ crosses the image of the lightcone of its support at $\underline{t}_{0}=-1 / t_{0}$. The hypersurface $\underline{t}_{0}=$ constant is used to place our initial data. For any point in $M^{-}=\bar{C}_{0}^{-} \cup\left\{\underline{t} \geqq \underline{t}_{0}\right\}$, the solution of the original problem is regular. Blow up, if there is any, should happen on $\mathscr{C}_{0}^{-}$. Fig. 2 should help one visualize all the actions of the inversion map.

\section{Regularity of the Initial Data in the Target Space}

To prove regularity of the solution at $\underline{t}_{0}=$ constant in $M^{-}$, we notice that $\phi$ is mapped smoothly onto this hypersurface, since it is the composition of two smooth functions. To show the regularity of the electromagnetic tensor we will use its null decomposition, since it is more convenient than the usual electric and magnetic decomposition. Let us introduce the null tetrad in $\mathrm{M}^{+}$,

$$
\begin{gathered}
l=\frac{1}{\sqrt{2}} d u, \quad \xi=r d \theta, \\
m=\frac{1}{\sqrt{2}} d v, \quad \chi=r \sin \theta d \phi,
\end{gathered}
$$

where $u$ and $v$ were defined earlier. 


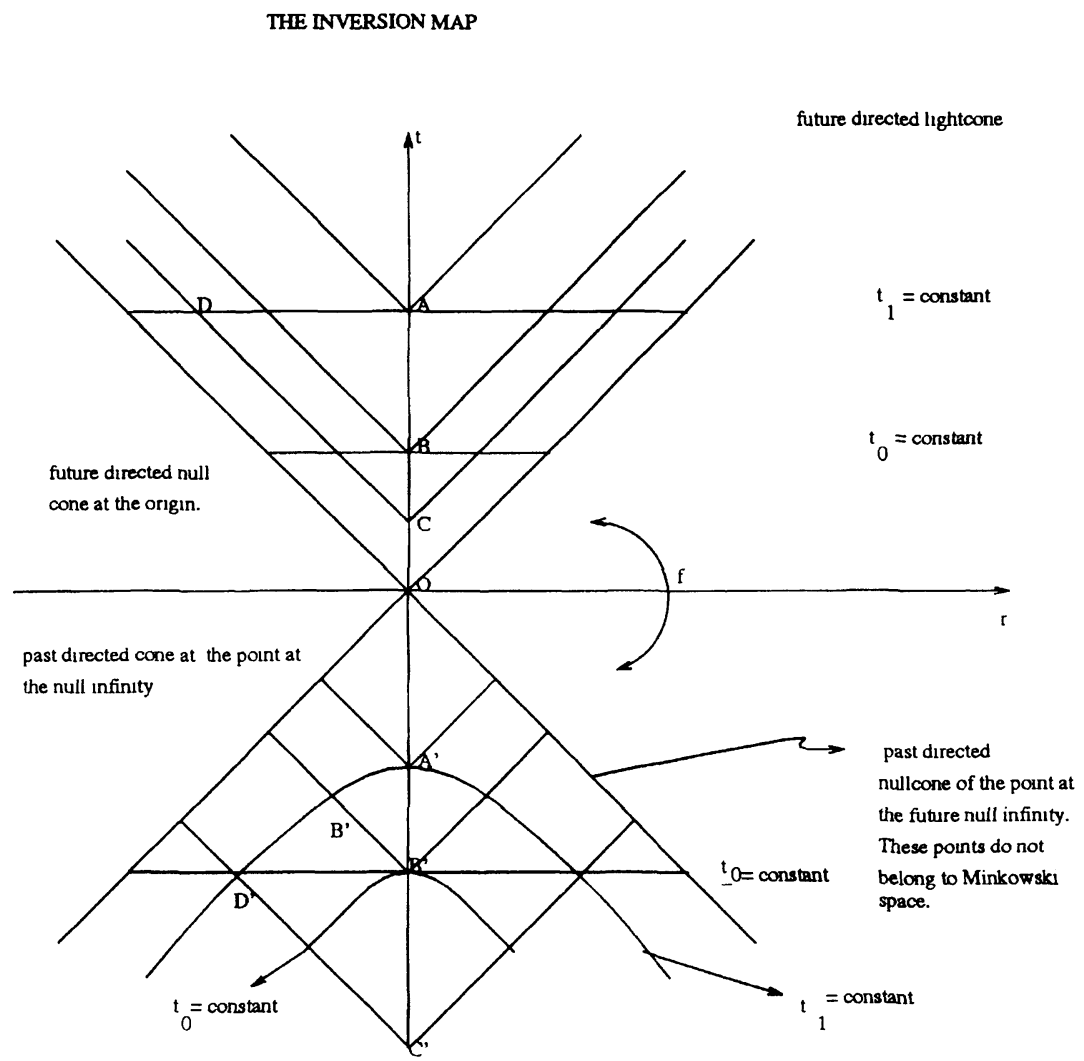

Fig. 2. The inversion map as seen from Minkowski space

In this basis we write $\mathbf{F}$ as

$$
\begin{aligned}
\mathbf{F}= & F_{l m} l \wedge m+F_{l \xi} l \wedge \xi+F_{m \xi} m \wedge \xi \\
& +F_{l \chi} m \wedge \chi+F_{m \chi} m \wedge \chi+F_{\xi \chi} \xi \wedge \chi .
\end{aligned}
$$

The correspondence of this decomposition with the usual electric and magnetic one is

$$
\begin{aligned}
\mathbf{E}_{\mathbf{r}} & =F_{l m}, \\
\mathbf{E}_{\xi}+\mathbf{B}_{\chi} & =F_{l \xi}, \\
\mathbf{E}_{\xi}-\mathbf{B}_{\chi} & =F_{m \xi}, \\
\mathbf{E}_{\chi}+\mathbf{B}_{\xi} & =F_{l \chi}, \\
\mathbf{E}_{\chi}-\mathbf{B}_{\xi} & =F_{m \chi}, \\
\mathbf{B}_{\mathbf{r}} & =F_{\xi \chi} .
\end{aligned}
$$

For any point $p \in S_{\phi(t)}$ the electric and magnetic fields are non-vanishing. By Lemma 1, the static solution outside $S_{\phi(t)}$ is such that the magnetic component is 
zero. In other words, outside $S_{\phi(t)}$ only the first 5 equations survive. $F_{\xi \chi}$ is zero there.

It is seen from Eqs. $(13,14)$ and Lemma 1 , that if $\left(x_{i}, t\right)$ are the coordinates of a point on a sphere of radius $|\mathbf{x}| \gg r_{t}$, where $r_{t}$ is the radius of $S_{\phi(t)}$. Then, for all $t$,

$$
\begin{aligned}
& E_{r}(x)=\int_{\mathscr{V}_{0}} \frac{\operatorname{Im}\left(\phi \overline{\partial_{0} \phi}\right)\left(x^{\prime \circ}\right)}{\left|\mathbf{x}-\mathbf{x}^{\prime}\right|^{3}}|\mathbf{x}| d^{3} x^{\prime}+o\left(|x|^{-3}\right), \\
& E_{\omega}(x)=\int_{\mathscr{V}_{0}} \frac{\operatorname{Im}\left(\phi \overline{\partial_{0} \phi}\right)\left(x^{\prime}\right)}{\left|\mathbf{x}-\mathbf{x}^{\prime}\right|^{3}}\left|\mathbf{x}^{\prime}\right| d^{3} x^{\prime}+o\left(|x|^{-4}\right),
\end{aligned}
$$

where $E$ means the function and not the vector (or form). $\mathscr{V}_{0}$ is the volume of the support of $\phi$. It follows that

$$
\begin{array}{cc}
\left|E_{r}(x)\right| \leqq \frac{Q}{|\mathbf{x}|^{2}}+o\left(|\mathbf{x}|^{-3}\right) & |\mathbf{x}| \rightarrow \infty \\
\left|E_{\omega}(x)\right| \leqq \frac{Q_{1}}{|\mathbf{x}|^{3}}+o\left(|\mathbf{x}|^{-4}\right) & |\mathbf{x}| \rightarrow \infty
\end{array}
$$

$Q$ and $Q_{1}$ are integrals that bound the charge and dipole moment respectively,

$$
\begin{aligned}
Q & =\int_{\mathscr{V}_{0}}\left|\operatorname{Im}\left(\phi \overline{\partial_{0} \phi}\right)\left(x^{\prime}\right)\right| d^{3} x^{\prime}, \\
Q_{1} & =\int_{\mathscr{V}_{0}}\left|\operatorname{Im}\left(\phi \overline{\partial_{0} \phi}\right)\left(x^{\prime}\right) \mathbf{x}^{\prime}\right| d^{3} x^{\prime} .
\end{aligned}
$$

To study what happens to the two-form $\mathbf{F}$, we need to study the map of the six functions $F_{l m}, F_{l \xi}, F_{m \xi}, F_{l \chi}, F_{m \chi}, F_{\xi \chi}$ and the pullback of the forms $l, m, \chi$ and $\xi$. We find the relation between null forms in $M^{-}$and the pullback of null forms of $M^{+}$ using that $f^{2}=i d$. First we define a set of null forms in $M^{-}$. These forms are (an underline denotes forms and functions in $M^{-}$)

$$
\begin{array}{ll}
\underline{l}=\frac{1}{\sqrt{2}} d \underline{u}, & \underline{\xi}=\underline{r} d \underline{\theta}, \\
\underline{m}=\frac{1}{\sqrt{2}} d \underline{v}, & \underline{\chi}=\underline{r} \sin \underline{\theta} d \underline{\phi} .
\end{array}
$$

The relations between null forms are

$$
\begin{aligned}
\underline{l}=-\underline{u}^{2} f^{*} l, & \underline{\xi}=\underline{u v} f^{*} \xi, \\
\underline{m}=-\underline{v}^{2} f^{*} m, & \underline{\chi}=\underline{u v} f^{*} \chi .
\end{aligned}
$$

The transformed form in the target space time is given by

$$
\begin{aligned}
\underline{\mathbf{F}}= & f^{*} \mathbf{F} \\
= & f^{*} F_{l m} f^{*} l \wedge f^{*} m+f^{*} F_{l \xi} f^{*} l \wedge f^{*} \xi+f^{*} F_{m \xi} f^{*} m \wedge f^{*} \xi \\
& +f^{*} F_{l \chi} f^{*} m \wedge f^{*} \chi+f^{*} F_{m \chi} f^{*} m \wedge f^{*} \chi+f^{*} F_{\xi \chi} f^{*} \xi \wedge f^{*} \chi,
\end{aligned}
$$


but we also have

$$
\begin{aligned}
& \underline{\mathbf{F}}=\underline{F}_{\underline{l} \underline{l}} \underline{l} \wedge \underline{m}+\underline{F}_{\underline{l} \underline{\xi}} \underline{l} \wedge \underline{\xi}+\underline{F}_{\underline{m} \underline{\xi}} \underline{m} \wedge \underline{\xi} \\
& +\underline{F}_{\underline{l} \underline{\chi}} \underline{m} \wedge \underline{\chi}+\underline{F}_{\underline{l} \underline{\chi}} \underline{m} \wedge \underline{\chi}+\underline{F}_{\underline{\xi} \underline{\underline{\xi}}} \underline{\xi} \wedge \underline{\chi} .
\end{aligned}
$$

We are now in position to establish the following.

Proposition 2. The map of the solution of Eqs. (5-7) in $\bar{C}_{0}^{+}$between $t_{0}$, the initial data hyperplane and $t_{1}$, given by Eq. (34), with the Coulomb initial data, is regular on the hypersurface $\left\{\underline{t}_{0}=\right.$ constant $\} \cap \bar{C}_{0}^{-}$.

Proof. The regularity in the region $\underline{t}_{0} \cap C_{0}^{-}$follows since the solution and the map are regular. One expects a problem only on the lightcone, i.e., in $\mathscr{C}_{0}^{-}$. Using the first term in the r.h.s. of Eq. (54), Eqs. (38) and (46) we have

$$
\begin{aligned}
f^{*} F_{l m} f^{*} l \wedge f^{*} m & =f^{*} E_{r} \frac{-1}{\underline{u}^{2}} \underline{l} \wedge \frac{-1}{\underline{v}^{2}} \underline{m} \\
& =f^{*} E_{r} \frac{1}{(\underline{u v})^{2}} \underline{l} \wedge \underline{m} .
\end{aligned}
$$

Using Eq. (46) and Eq. (55) we define

It follows that

$$
\underline{F}_{\underline{l m}}=f^{*} E_{r} \frac{1}{(\underline{u v})^{2}} .
$$

$$
\begin{aligned}
\underline{F}_{l m} & \leqq Q f^{*}\left(\frac{1}{r^{2}}\right) \frac{1}{(\underline{u v})^{2}} \\
& \leqq Q\left(\frac{\underline{u v}}{\underline{r}}\right)^{2} \frac{1}{(\underline{u v})^{2}} .
\end{aligned}
$$

Coming from the second term we have

$$
\begin{aligned}
f^{*} F_{l \xi} f^{*} l \wedge f^{*} \xi & =f^{*} E_{\omega} \frac{-1}{\underline{u}^{2}} \underline{l} \wedge \frac{-1}{\underline{u v}} \underline{\xi} \\
& =f^{*} E_{\omega} \frac{1}{\left(\underline{u^{3}} \underline{v}\right)} \underline{l} \wedge \underline{\xi} .
\end{aligned}
$$

Again using Eq. (47) and Eq. (55)

then

$$
\underline{F}_{\underline{l} \underline{\xi}}=f^{*} E_{\omega} \frac{1}{\left(\underline{u}^{3} \underline{v}\right)},
$$

$$
\begin{aligned}
\underline{F}_{\underline{l} \underline{\xi}} & \leqq Q_{1} f^{*}\left(\frac{1}{r^{3}}\right) \frac{1}{\left(\underline{u}^{3} \underline{v}\right)} \\
& \leqq Q_{1}\left(\frac{\underline{u} v}{\underline{r}}\right)^{3} \frac{1}{\left(\underline{u}^{3} \underline{v}\right)}
\end{aligned}
$$


Proceeding in this fashion we obtain all the other bounds. Finally

$$
\begin{array}{r}
\left|\underline{F}_{\underline{l} \underline{\underline{\xi}}}\right|,\left|\underline{F}_{\underline{l \underline{x}}}\right| \leqq Q_{1} \frac{\underline{v}^{2}}{\underline{r}^{3}}, \\
\left|\underline{F}_{\underline{l m}}\right| \leqq Q \frac{1}{\underline{r}^{2}}, \\
\left|\underline{F}_{\underline{\underline{m}} \underline{\xi}}\right|,\left|\underline{F}_{\underline{\underline{x}} \underline{\underline{x}}}\right| \leqq Q_{1} \frac{\underline{u}^{2}}{\underline{\underline{r}}^{3}},
\end{array}
$$

and $\underline{F}_{\underline{\xi} \underline{\underline{\chi}}}=0$. One of the advantages of the null decomposition is that conformal diffeomorphisms map null components into the same null components.

On $\mathscr{C}_{0}^{-}, \underline{v}=0$ and both $\underline{u}$ and $\underline{r}$ are finite and bounded away from zero for $\underline{t}=\underline{t}_{0}$. Equation (54) and the bounds above imply that the form $\underline{\mathbf{F}}$ is well defined on the linhtcone.

The field is real analytic in the region outside $S_{\phi(t)}$. The multipole expansion

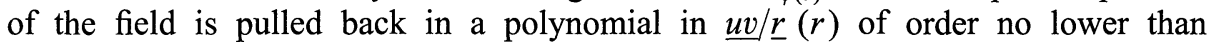
two. Taking into account the bounds obtained earlier and the fact that the func-

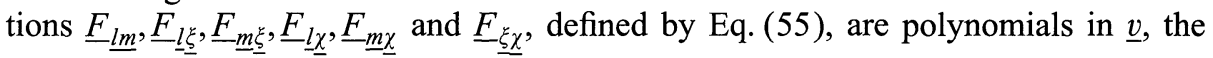
extension to the boundary is made smoothly ${ }^{2}$.

Next, we prove that the gauge field $\tilde{\mathbf{A}}$ is regularly mapped onto $\left\{\underline{t}_{0}=\right.$ constant $\} \cap$ $\bar{C}_{0}^{-}$. Similarly to what was done with the tensor field $\mathbf{F}$, we write the null decomposition of $\tilde{\mathbf{A}}$ as

$$
\tilde{\mathbf{A}}=\tilde{A}_{l} l+\tilde{A}_{m} m+\tilde{A}_{\xi} \xi+\tilde{A}_{\chi} \chi .
$$

The gauge is such that $\tilde{A}_{m}=0$. Outside $S_{\phi(t)}$ the other components of $\tilde{A}_{m}$ have decay no worse than $1 / r$ because the field decays no worse than $1 / r^{2}$ (see Eq. (12)). Using the relationship between the null components in the two spaces (Eqs. (52),(53)) and that $f$ maps null components in null components we get

$$
f^{*} \tilde{\mathbf{A}}=f^{*} \tilde{A}_{l} f^{*} l+f^{*} \tilde{A}_{\xi} f^{*} \xi+f^{*} \tilde{A}_{\chi} f^{*} \chi,
$$

because $\tilde{A}_{m}=0$. Since $f^{*}(1 / r)=\underline{u v} / \underline{r}$ and the pullback of all the forms used in Eq. (68) have at most $\underline{v}$ in the denominator, it follows that the pullback of $\tilde{\mathbf{A}}$ is smooth. The only component that could have caused a headache was eliminated in our selection of the gauge. The pullback of $m$ explodes as $\underline{v}^{-2}$, much faster than decay of $\underline{\tilde{A}}_{m}, \underline{v}$.

Proving the smoothness of the scalar field is immediate, since it is supported in $\left\{\underline{t}_{0}=\right.$ constant $\} \cap C_{0}^{-}$in $M^{-}$.

As a corollary, we can speak of an initial value problem in $M^{-}$, with smooth initial data given on the hypersurface $\underline{t}_{0}=$ constant. Changing back to the temporal gauge in the target space is of no difficulty. Using Theorem 1, one establishes the global existence of the solution for the Cauchy problem in $\mathrm{M}^{-}$.

\footnotetext{
there

${ }^{2}$ the boundary lies outside the original space, the function is extended to take smooth values
} 


\section{Time Decay of the Solution}

We have established $L^{\infty}\left(\mathbb{R}^{3}\right)$ bounds for Maxwell and scalar fields. These bounds are established for a compact in time interval, therefore, we have a $L^{\infty}\left(M^{-}\right)$bound. To see what kind of time decay these bounds imply, we send all the forms and the scalar function from the target to the original space. First, we deal with the scalar field. The uniform bounds obtained in $M^{-}$imply that $\Phi$ is uniformly bounded. Using $\phi=\Omega \Phi$ and $\Omega=-s^{-2}$, it follows that

$$
|\Phi|=|u v||\phi| \leqq c
$$

or,

$$
|\phi| \leqq c \frac{1}{(t+r)(t-r)} \quad t>r .
$$

All points inside the outward future directed lightcone of $S_{\phi(t)}$ have both $u$ and $v$ strictly greater than zero and $t-r>r_{0}+1$.

To obtain the time decay of the null components of the Maxwell field, we use that all null components of the Maxwell field in $M^{-}$are bounded by a constant (they are uniformly bounded). This means that we can write a two-form $\mathbf{Q}$ in $M^{-}$ that bounds the Maxwell fields. The pullback of the form $\mathbf{Q}$ bounds the Maxwell field in $M^{+}$. This form is

$$
\mathbf{Q}=c(\underline{l} \wedge \underline{m}+\underline{l} \wedge \underline{\xi}+\underline{m} \wedge \underline{\xi}+\underline{m} \wedge \underline{\chi}+\underline{m} \wedge \underline{\chi}+\underline{\xi} \wedge \underline{\chi}) .
$$

Pulling back $\mathbf{Q}$, using that it bounds the Maxwell fields and

$$
\begin{array}{cl}
l=-u^{2} f^{*} \underline{l}, & \xi=u v f^{*} \underline{\xi}, \\
m=-v^{2} f^{*} \underline{m}, & \chi=u v f^{*} \underline{\chi},
\end{array}
$$

one obtains the following time decay for null components of tensor fields in terms of $t$ and $r$, with $t-r>r_{0}+1$,

$$
\begin{aligned}
\left|F_{l \xi}\right|,\left|F_{l \chi}\right| & \leqq c \frac{1}{(t-r)^{3}(t+r)}, \\
\left|F_{m \xi}\right|,\left|F_{m \chi}\right| & \leqq c \frac{1}{(t-r)(t+r)^{3}}, \\
\left|F_{l m}\right|,\left|F_{\xi \chi}\right| & \leqq c \frac{1}{(t-r)^{2}(t+r)^{2}} .
\end{aligned}
$$

Adding everything up, we have achieved proving the following theorem.

Main Theorem The Cauchy problem for the Maxwell-Klein-Gordon conformally covariant system $(5,7)$ with Coulomb initial data, (conditions $h 1, h 2$ and h3) has a unique solution, defined globally in Minkowski spacetime by Theorem 1. Furthermore, using Proposition 2 and Theorem 1 we show that the solution has the decay given by Eqs. (70), (74-76). 


\section{References}

1. Eardley, D., Moncrief, V.: The Global Existence of Yang-Mills-Higgs Fields in 4-dimensional Minkowski Space. Commun. Math. Phys. 83, 192-212 (1982)

2. Eardley, D., Moncrief, V.: The Global Existence of Yang-Mills-Higgs Fields in 4-dimensional Minkowski Space. Commun. Math. Phys. 83, 171-191 (1982)

3. Choquet-Bruhat, Y., Morette, C.W., Bleick, M.D.: Analysis, Manifolds and Physics. Amsterdam: North Holland, revised edition, 1987

4. Choquet-Bruhat, Y., Christodoulou, D.: Existence of Global Solutions of the Yang-Mills, Higgs and Spinor Field Equations in $3+1$ Dimensions. Ann. Sci. Ec. Norm. Sup, $4^{e}$ serie, $481-500$ (1981)

5. Christodoulou, D.: Solutions Globales des Equations de Champs de Yang-Mills. C.R. Acad. Sci. Paris 293, Series A, 39-41 (1981)

Communicated by S.-T. Yau 
